\title{
Digital Compensation of Residual Pump Dithering in Optical Phase Conjugation of High-Order QAM
}

\author{
T. T. Nguyen, ${ }^{1,}$ S. Boscolo, ${ }^{1, *}$ A. A. I. Ali,${ }^{1}$ M. Tan, ${ }^{1}$ S. Sygletos, ${ }^{1}$ S. Takasaka, ${ }^{2}$ R. \\ Sugizaki, ${ }^{2}$ and A. D. Ellis ${ }^{1}$ \\ ${ }^{1}$ Aston Institute of Photonic Technologies, Aston University, Aston Triangle, Birmingham B4 7ET, UK \\ ${ }^{2}$ Furukawa Electric Co. Ltd., 6 Yawata-kaigandori, Ichihara, Chiba, 290-8555, Japan \\ ${ }^{\dagger}$ Now with Infinera PA, 7360 Windsor Dr, Allentown, PA 18106, USA. * s.a.boscolo@aston.ac.uk
}

\begin{abstract}
We develop a new two-stage digital scheme to suppress the phase distortion due to residual pump dithering in the dual-pump fibre-based optical phase conjugation of high-order (64/256) QAM signals. We show more than 5-dB SNR improvement relative to conventional phase-noise compensation at high pump-phase mismatch levels. (C) 2021 The Author(s)
\end{abstract}

\section{Introduction}

Optical phase conjugation (OPC) is gaining increasing attention as an in-line all-optical signal processing technique that provides transparent dispersion and nonlinearity compensation when deployed symmetrically along the transmission link [1]. However, in fibre-based OPC devices relying on four-wave mixing, the pump phase modulation used to suppress stimulated Brillouin scattering introduces periodic phase distortions on the conjugated signal (idler), which are detrimental to phase-modulated signals. Even in dual-pump configurations in which the two pumps are modulated using complementary phase patterns [2], deviations from the ideal case commonly exist in practice, and in fact are one of the main performance limiting factors of the OPC. Traditional digital signal processing (DSP) techniques for the compensation of the phase noise (PN) induced by laser non-zero spectral width in coherent optical systems [3,4] as standalone cannot efficiently counteract the deterministic effects of imperfections in the dithering scheme of OPC systems where the idler phase modulation is strong, because of either their decision-directed operation [3] or the requirement of a relatively constant PN evolution over a long time window [4]. The frequency-domain peak searching ( $m$ th-power based) algorithm demonstrated in [5] to estimate the parameters of the pump dithering from the phase of the idler symbols before carrier phase recovery performs poorly with non-uniform phase-distribution signals, therefore it is not suitable to high-order modulation formats. Further, the usability of this method with dual-pump schemes is questionable because the residual phase modulation is low and the spectrum has many components.

In this paper, we develop a new method for compensating the phase distortion caused by deviations from ideal pump counter-dithering in the OPC of high-order quadrature-amplitude modulation (QAM) signals, which we implement using a two-stage design. After conventional PN compensation, in the second stage for each dominant spectral component (corresponding to the known phase-modulation frequencies) we perform a least squared error fit to the required tone amplitude and angle. The proposed scheme is verified numerically and experimentally with $28 \mathrm{Gbaud}$ dual-polarisation 64- and 256-QAM signals. The large improvement in signal-to-noise ratio (SNR) achieved by the second stage compared to the conventional PN compensation stage indicates that it is possible to relax the requirement of pump counter-phasing calibration in the OPC implementation.

\section{System Description and Two-Stage PN Compensation Method}

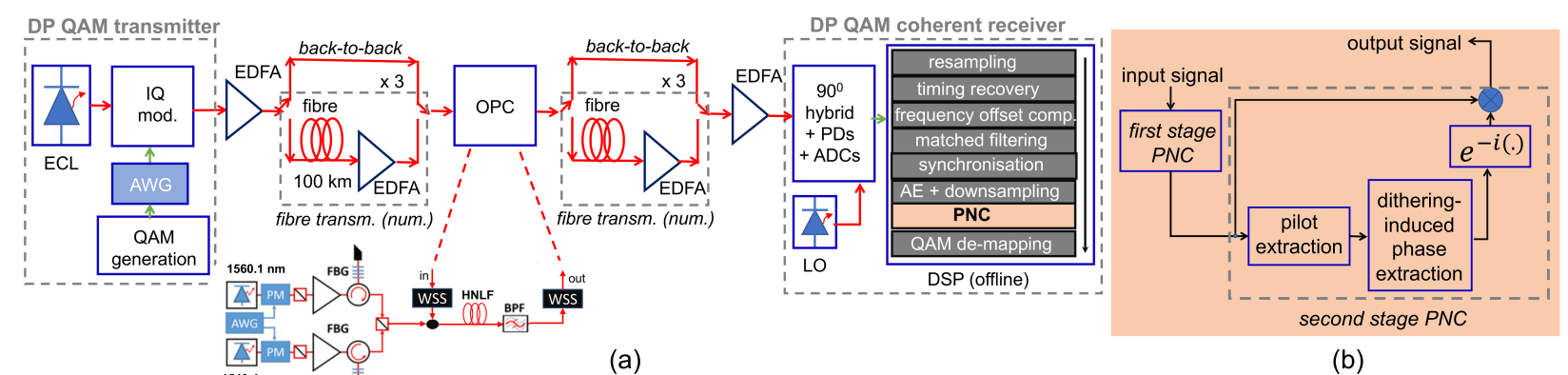

Fig. 1. (a) Experimental setup of the OPC-assisted dual-polarisation 28 Gbaud 64/256-QAM system equipped with dual-stage PN compensation. Also shown is the simulated inline-EDFA transmission configuration. (b) Block diagram of the two-stage PN compensation algorithm implementation.

The experimental setup of the OPC-assisted 28-Gbaud dual-polarisation 64/256-QAM system is shown in Fig. 1a. The details of the QAM transmitter and coherent receiver are given in [6]. The transmitted optical signal is conjugated through a polarisation-insensitive dual-band OPC with orthogonally polarised pumps spectrally located at $1540.4 \mathrm{~nm}$ and $1560.1 \mathrm{~nm}$, and with laser linewidth $\sim 30 \mathrm{kHz}$ [7]. One signal band only is used in this experiment. Two radio-frequency (RF) tones at frequencies $f_{1}=60 \mathrm{MHz}$ and $f_{2}=600 \mathrm{MHz}$ are generated by an arbitrary waveform generator (AWG) and used to independently phase-modulate the pump lasers via optical phase 
modulators. The amplitudes and phases of the RF tones for each phase modulator are first adjusted to minimise the transfer of phase modulation from the pumps to the generated idler. Then, we intentionally increase the pumpphase mismatch up to $10^{\circ}$ by tuning the phase of one of the RF tones. The highly nonlinear fibre is 100-m long, and the nominal values of the attenuation coefficient, Kerr-nonlinearity coefficient, zero-dispersion wavelength, and dispersion slope are $\alpha=1.2 \mathrm{~dB} / \mathrm{km}, \gamma=21.4(\mathrm{~W} \cdot \mathrm{km})^{-1}, 1550 \mathrm{~nm}$, and $0.041 \mathrm{ps} /\left(\mathrm{nm}^{2} \cdot \mathrm{km}\right)$, respectively. In our numerical model of OPC, we consider a dual-pump unidirectional system with parameters slightly adjusted from the nominal values in order to match the experimentally observed conversion efficiency. The phase modulations of the pump waves are modelled as $\phi_{m i}(t)=\int \mathrm{d} \tau h(t-\tau)\left[\alpha_{1} f_{i}(\tau)+\alpha_{2} f_{i}(\tau)^{2}+\alpha_{3} f_{i}(\tau)^{3}\right], i=1,2$, where $f_{1}(t)=(m+\delta m)\left[\cos \left(2 \pi f_{1} t+\delta \theta\right)+\cos \left(2 \pi f_{2} t+\delta \theta\right)\right], f_{2}(t)=m\left[-\cos \left(2 \pi f_{1} t\right)-\cos \left(2 \pi f_{2} t\right)\right]$, the $\alpha$ 's are the coefficients of the polynomial response of the RF power amplifiers, $h(t)$ is the impulse response of the low-pass filters that filter the output waveforms from the amplifiers, $m$ is the modulation index, the modulation-index mismatch $\delta m$ represents possibly different modulation indices, and the pump-phase mismatch $\delta \theta$ accounts for a non-ideal phase shift. It is easy to see that the pump-phase modulation contribution to the idler phase only vanishes for ideal counter-phasing, $\delta m=\delta \theta=0$, under the assumption of linear amplifier's response. This is difficult to achieve in practice. Here, to obtain a good match to the experimentally observed residual dither, we use $m=1.3 \mathrm{rad}, \delta m=0.01 \mathrm{rad}, \alpha_{1}=1$, $\alpha_{2}=0.007, \alpha_{3}=0.005$, and we vary the pump phase-mismatch parameter $\delta \theta$ to control the deviation of the pump phases from ideal counter-phasing. After coherent detection, a standard DSP procedure for data recovery $[6,8]$ is implemented offline. The signal is down-sampled to 1 sample per symbol before being fed into the proposed two-stage PN compensation module. At the output of this module, the pilot symbols are removed before QAM de-mapping. The recovered QAM symbols together with the transmitted symbols are used to measure an effective SNR defined as $\mathrm{SNR}=\mathbb{E}_{k}\left[|x[k]|^{2}\right] / \mathbb{E}_{k}\left[|\hat{y}[k]-x[k]|^{2}\right]$, where $x[k]$ and $\hat{y}[k]$ are the respective transmitted and received QAM symbols at the time instance $k$ and $\mathbb{E}$ is the expectation operator.

Assuming perfect timing recovery, ideal synchronisation and zero frequency-offset, the phase-distorted signal at the input to the PN compensation unit can be written in the form $y[k]=x[k] \exp \left[i\left(\delta \phi[k]+\phi_{m}[k]\right)\right]+\varepsilon[k]$, where $\varepsilon[k]$ is the additive white Gaussian noise present in the system. The first phase term $\delta \phi[k] \sim \mathscr{N}\left(0,2 \pi \delta v T_{s}\right)$ represents the Wiener random laser PN, where $\delta v$ is the combined spectral linewidth of the system (total linewidth of transmit, receive and pump lasers), and $T_{s}$ is the symbol period. The second phase term $\phi_{m}[k]=\phi_{m 1}[k]+\phi_{m 2}[k]$ represents the deterministic phase distortion generated by imperfect pump counter-phasing. The aim of our compensation algorithm is to estimate and remove this phase component. To this end, we apply a two-stage procedure (Fig. 1b). The first stage is a conventional pilot-aided phase estimation algorithm [4], in which 8 pilots in each block are used for noise averaging. After the first stage, the phase distortion due to both random laser PN and the effect of the residual pump dithering is partly removed from the input signal. We can therefore focus the second PN compensation block on the dominant residual dithering components, which occur at the original dithering drive frequencies. Indeed, the intermodulation products are sufficiently numerous to be treated as additive noise, and we therefore assume that the first stage can compensate for these. Hence in the second block, we determine the optimum amplitudes $a_{i}$ and angles $\theta_{i}$ of the tone signals. For each tone, a blind grid search $(10 \times 10)$ is performed with a step-size of $0.02 \mathrm{rad}$ and $0.63 \mathrm{rad}$ for the amplitude and angle, respectively. The number of preset values and step sizes are chosen to balance the algorithm's performance with its complexity. Inside each iteration, a test cosine wave is constructed, which is then used to phaserotate the extracted pilot symbols. After the two-dimensional sweeping, the optimum amplitudes and angles of the cosine waves are determined by searching the minimum sum of distance values of the test symbols to the original pilots. Finally, the optimum phase modulation is used to rotate the signal after the first stage of PN compensation. The overall computational complexity of the algorithm can be estimated as $2 N_{a} N_{\theta}(1024+512)$, where factor $2, N_{a}$ and $N_{\theta}$ represent the respective numbers of frequencies and preset values of the tone's amplitude and angle that are evaluated, and 1024 and 512 are the numbers of real-valued multipliers required for the construction of the test symbols and the error calculation, respectively, in each for run.

\section{Results and Discussion}

Figure 2a shows the performance of the proposed two-stage PN compensation scheme in terms of effective SNR for varying pump-phase mismatch $\delta \theta$. In this optical back-to-back experiment, the OPC transceiver is set to operate at an optical SNR of $\sim 36 \mathrm{~dB}$ under ideal pump counter-phasing $\left(\delta \theta=0^{\circ}\right)$. We observe that the performance of the conventional PN compensation stage degrades significantly under the impact of imperfect pump counter-phasing for both 64- and 256-QAM: more than 1-dB SNR penalty is observed at a pump-phase mismatch of only $3^{\circ}$. In contrast, the proposed two-stage scheme can tolerate the whole range of pump-phase mismatches being tested under the operating condition of less than 1-dB SNR penalty. At $\delta \theta=8^{\circ}$ the two-stage scheme has a SNR improvement of $\sim 5 \mathrm{~dB}$ relative to the one-stage scheme for both QAM formats, with this enhancement approaching $7 \mathrm{~dB}$ at $\delta \theta=10^{\circ}$. The majority of the wrong symbols in the signal constellations after the first compensation stage are located at the outer constellation rings, reflecting the fact that the outer symbols are more susceptible to PN than the inner ones (Fig. 2b). In contrast, almost all phase errors vanish by use of the two-stage approach. Further, we observe that the performance of the compensation schemes evaluated by numerical simulation of the model system generally matches very well the performance calculated from experimental data, although this agreement is slightly worse in the 256- 
QAM case due to the more severe impact of the transceiver's implementation. Shown in Fig. 2b is the effective SNR as a function of the optical SNR (OSNR) when the proposed PN compensation method is applied to the two QAM formats being studied under different pump-phase mismatch levels. We can see that the effective SNR achieved by the conventional PN compensation stage increases at a slower rate with the OSNR and deviates from a linear increase at lower OSNR levels with increasingly stronger residual pump dithering. Conversely, the performance of the second stage is relatively insensitive to the pump-phase mismatch level, thereby leading to an effective SNR gain over the first stage which increases with higher OSNR and stronger residual pump dithering.
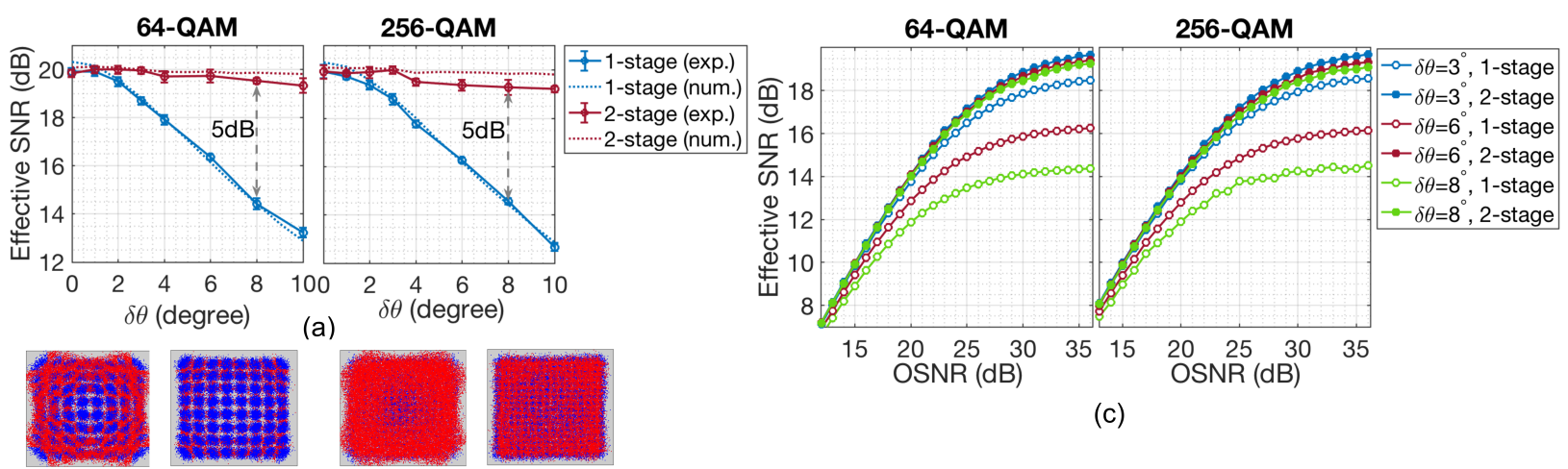

(b)

Fig. 2. Performance of the proposed PN compensation approach with 64- and 256-QAM systems in backto-back setup. (a) Effective SNR versus pump-phase mismatch after one- and two-stage PN compensation at $\mathrm{OSNR}=36 \mathrm{~dB}$. The experimental and numerical results are represented by solid and dotted curves, respectively. (b) Constellation diagrams at $\delta \theta=8^{\circ}$ for one- and two-stage PN compensation. (c) Effective SNR versus OSNR after one- and two-stage PN compensation at $\delta \theta=3^{\circ}, 6^{\circ}$, and $8^{\circ}$.

Taking advantage of the good agreement between the numerical and experimental results, we use numerical simulations to study the behaviour of the proposed two-stage PN compensation scheme under an inline erbium-doped fibre amplifier (EDFA) transmission configuration with mid-link OPC. The fibre channel includes 6 identical spans, each of $100-\mathrm{km}$ standard single-mode fibre $\left(\alpha=0.2 \mathrm{~dB} / \mathrm{km}\right.$, group-velocity dispersion $\left.=-21.7 \mathrm{ps}^{2} / \mathrm{km}, \gamma=1.3(\mathrm{~W} \cdot \mathrm{km})^{-1}\right)$ followed by an EDFA (noise figure $=6 \mathrm{~dB}$ ) to compensate for the fibre loss. The results in Fig. 3 highlight that the second-stage of PN compensation always outperforms the first stage, and brings about a SNR advantage at the optimum launched power of $>0.5 \mathrm{~dB},>1 \mathrm{~dB}$ and $\sim 2 \mathrm{~dB}$ for low, moderate and severe residual pump dithering, respectively. However, the benefit of the method is significantly degraded with respect to the back-to-back configuration. We ascribe this to the impact of fibre dispersion in the second half of the link in an analogous way to equaliser-enhanced PN. A modification of the PN compensation algorithm to address this issue is currently being investigated.

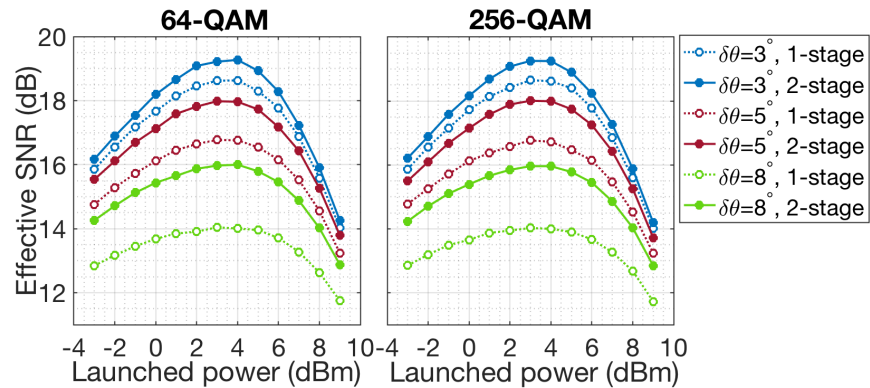

Fig. 3. Effective SNR versus launched power after one- and two-stage PN compensation at $\delta \theta=3^{\circ}, 5^{\circ}$, and $8^{\circ}$ for 64- and 256-QAM 600-km transmission.

\section{Conclusion}

We have developed a new two-stage DSP scheme to compensate the phase distortion induced by deviations from ideal pump counter-phasing for dual-pump OPC of high-order QAM signals. We have demonstrated numerically and experimentally that the proposed approach achieves large SNR improvement relative to conventional PN compensation when it is used with 64/256-QAM signals in the presence of severe imperfections in the pump-modulation scheme. We believe that this technique may firstly allow the applied pump dithering to be increased, improving the conversion efficiency and secondly, reduce residual dithering penalties for OPC systems with lower optical SNR penalties. The adaptation of our PN compensation algorithm to OPC-assisted transmission setups is a subject of ongoing research.

Acknowledgements: This work was supported by the UK EPSRC - Grants EP/S003436/1, EP/S016171/1 and EP/R035342/1.

\section{References}

1. A. D. Ellis et al., Adv. Opt. Photon. 9, 429-503 (2017).

2. M.-C. Ho et al., J. Lightwave Technol. 20, 469-476 (2002).

3. T. Pfau et al., J. Lightwave Technol. 27, 989-999 (2009).
4. S. Randel et al., IEEE Photon. Technol. Lett. 22, 1288-1290 (2010).

5. R. Elschner et al., Proc. ECOC, P3.17 (2010).

6. T. T. Nguyen et al., J. Lightw. Technol. 39, 388-399 (2021).

7. M. A. Z. Al-Khateeb et al., Opt. Express 26, 23960-23969 (2018).

8. S. J. Savory, Opt. Express 16, 804-817 (2008). 